\title{
ESTUDO DA INFLUÊNCIA DA TEMPERATURA DO ELETRODO NA QUALIDADE DA SOLDA PARA A UNIÃO DE CHAPAS NO PROCESSO DE SOLDAGEM POR RESISTÊNCIA ELÉTRICA (RSW)*
}

Gabriel Mendes de Almeida Carvalho ${ }^{1}$ Fagner Guilherme Ferreira Coelho ${ }^{2}$ Vinícius Nascimento e Souza ${ }^{3}$ Alexandre Queiroz Bracarense ${ }^{4}$

\section{Resumo}

O processo de soldagem de pontos por resistência elétrica é uma técnica econômica e adequada para a união de chapas, pela alta velocidade e adaptabilidade, torna-se um método amplamente difundido para a produção em massa. É utilizado principalmente em carrocerias de automóveis por apresentar elevada eficiência e qualidade. Este artigo analisa as diferenças apresentadas nas microestruturas de pontos de solda produzidos através do controle da refrigeração do eletrodo. Para garantir a qualidade de uma junta soldada e aumentar a vida útil do eletrodo é importante que os parâmetros empregados na soldagem sejam bem ajustados, para que o ponto de solda também apresente características que irão garantir uma boa performance do produto final. O objetivo deste trabalho é fazer a comparação da qualidade dos pontos de solda produzidos, além de avaliar a estrutura formada em diferentes temperaturas do eletrodo no momento da soldagem.

Palavras-chave:Microestrutura; RSW; Solda ponto; Controle de temperatura.

\section{THE INFLUENCE OF TEMPERATURE ON THE QUALITY OF THE SPOT WELD FOR JOINING SHEETS IN THE PROCESS RESISTANCE SPOT WELD (RSW)}

\begin{abstract}
The resistance spot welding is an economical and suitable technique for joining plates, the high speed and adaptability, it is a widespread method for mass production. It is mainly used in car bodies to provide high efficiency and quality. This paper analyzes the differences presented in the microstructures of weld spot produced by the electrode cooling control. To ensure the quality of a welded joint and extend the life of the electrode is important that the parameters used in welding are well adjusted to the welding spot also has characteristics that will ensure good performance of the final product. The objective of this work is to test the quality of produced welding spots, and to evaluate the structure formed at different temperatures of the electrode at the time of welding.

Keywords: Microstructure; RSW; Spot weld; Temperature control.

1 Graduando em Engenharia Mecânica, Universidade Federal de Minas Gerais (UFMG), Belo Horizonte, Minas Gerais, Brasil.

2 Engenheiro Mecânico, Mestre em Engenharia Mecânica, Departamento de Engenharia Mecânica, Universidade Federal de Minas Gerais, Belo Horizonte, Minas Gerais, Brasil.

3 Graduando em Engenharia Mecânica, Universidade Federal de Minas Gerais (UFMG), Belo Horizonte, Minas Gerais, Brasil.

4 Físico, Professor, Pesquisador, PhD - Universidade Federal de Minas Gerais (UFMG), Departamento de Engenharia Mecânica, Belo Horizonte, Minas Gerais, Brasil.
\end{abstract}


Solda por resistência elétrica é um processo de soldagem que requer a aplicação de pressão e calor para obter a união de duas chapas, ou seja, as peças serão unidas quando pressionadas uma contra a outra por meio de eletrodos, permitindo a passagem de corrente elétrica. Tem-se a pressão através da aplicação de força pela pinça que também é o suporte para os eletrodos, o aquecimento se dá devido ao efeito Joule $\left(Q=R . i^{2} . t\right)$. A forma mais simples do processo de soldagem por pontos é representada na Figura 1.

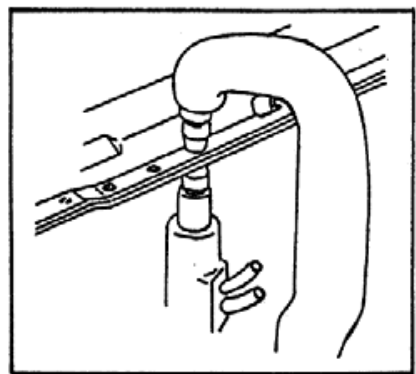

Figura 1. Processo de Soldagem a PontoFonte: [1]

A temperatura que devemos aplicar nas chapas deve ser suficiente para que ocorra a fusão localizada sem comprometer a estrutura do metal. O tempo necessário para que a corrente possa fluir e realizar a solda deve ser tal que quanto maior for seu valor, maior será o diâmetro do weldnugget até o limite do diâmetro do eletrodo utilizado. A compressão que as chapas estarão submetidas devido a força dos eletrodos afetam a qualidade da solda, pois altera a resistência na face de contato entre as chapas [2].

As regiões aquecidas no processo podem ser separadas em isotermas, de acordo com a forma com que afeta a estrutura do ponto soldado [3]. A Figura 2 ilustra estas isotermas separando as em regiões (I - zona dendrítica e II Zona afetada pelo calor).

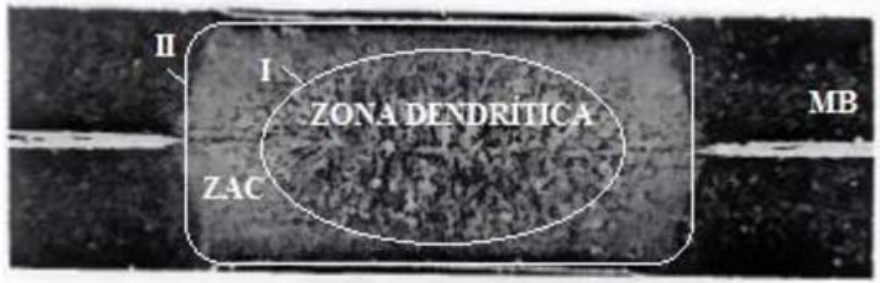

Figura 2. Aplicação de um ponto de solda evidenciando três regiões distintas:

I- Zona dendrítica; II- Zona afetada pelo calor (ZAC); Metal de base (MB) .Fonte [3]

O principal uso da RSW (Resistance Spot Welding) é o baixo custo, alta velocidade para a união de peças metálicas sobrepostas, além de não necessitarem desmontagem para a união das chapas. A Figura 3 mostra os principais parâmetros geométricos do ponto de solda, mostrando a profundidade e largura da indentação que serão avaliadas nesse trabalho. 


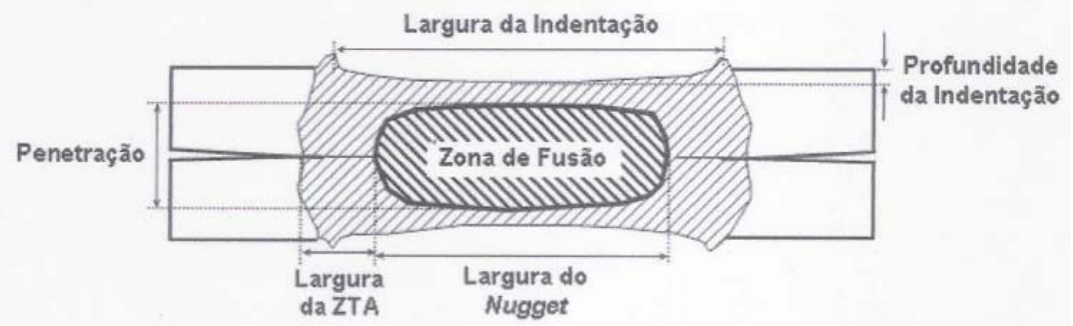

Figura3. Detalhes das regiões do processo de soldagem a ponto Fonte [6].

Os eletrodos que possuem pequeno diâmetro (normalmente de 3 a $8 \mathrm{~mm}$ ), tem como função conduzir a corrente elétrica para execução da solda, além de transmitir força, e manter alinhadas as peças. Elevados níveis de correntes, acabamento superficial e principalmente a deficiência da refrigeração nos eletrodos promovem redução da vida útil dos mesmos [5].

O sistema de resfriamento de eletrodos, segundo [6], deve ter especial atenção na distribuição de temperaturas quando refrigerados a água. A refrigeração do eletrodo aliada à condução de calor pelas chapas são as grandes responsáveis pela diminuição no rendimento do processo. A distância entre o tubo e a ponta (capa) do eletrodo é muito importante para o resfriamento do mesmo. A vazão de água recomendada para os eletrodos deve ser de no mínimo $4 \mathrm{~L} / \mathrm{min}$ para chapas de aço sem revestimento, e de $6 \mathrm{~L} / \mathrm{min}$ para chapas com revestimento. A temperatura da água deve ser controlada na entrada do sistema de resfriamento para que não haja influência no processo [7]. A Figura 4 mostra a representação esquemática da refrigeração do eletrodo.

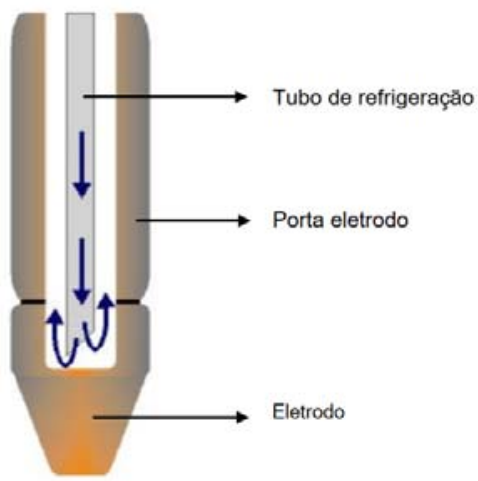

Figura 4. Representação de um sistema de refrigeração do eletrodo de um equipamento de soldagem por resistência elétrica Fonte[10].

O presente trabalho faz a comparação dos pontos de solda produzidos com eletrodos a diferentes temperaturas no momento da soldagem. Utilizando um sistema de refrigeração com controle de temperatura, os eletrodos de cobre foram mantidos a uma temperatura constante logo antes do inicio da soldagem procurando levantar a sua influência na geometria do ponto e na qualidade da solda. Com isso tem-se uma nova variável do processo e a definição de uma temperatura de soldagem ideal, atendendo a crescente demanda de confiabilidade e segurança de componentes soldados, principalmente de automóveis. 


\section{MATERIAIS E MÉTODOS}

\subsection{Materiais}

Para o desenvolvimento deste trabalho para avaliar o comportamento do campo de temperatura durante a soldagem foram realizados testes para 03 diferentes valores $\left(30^{\circ} \mathrm{C}, 40^{\circ} \mathrm{C}, 50^{\circ} \mathrm{C}\right)$, e para cada um deles foram realizadas 05 medidas, dos quais foram considerados os 03 melhores resultados. Com isso, tem-se um total de 09 corpos de prova, e estes conforme apresentado na Figura 5 segue a norma AWS D8.9.

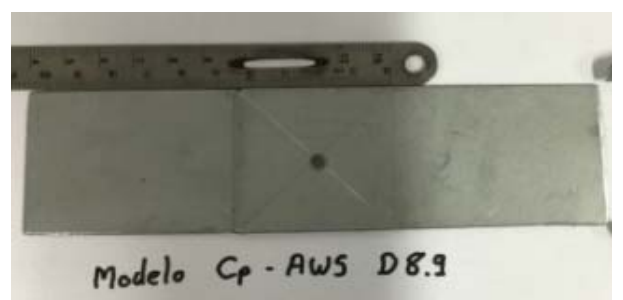

Figura 5. Corpo de prova produzido no trabalho.Fonte: Os Autores.

O corpo de prova produzido é composto por duas chapas galvanizadas de aço baixo carbono, com espessura de $0,80 \mathrm{~mm}$ e revestimento mínimo de $\mathrm{Zn}$ de $10,0 \mu \mathrm{m}$ pelo processo de revestimento por imersão.

Os experimentos foram realizados em uma máquina de soldagem por pontos comercial do fabricante FASE, modelo X/4-200, composta por pinças suspensas e com acionamento pneumático por gatilho apresentada na Figura 6. Foi instalada uma bomba de vazão igual a $12 \mathrm{~L} / \mathrm{min}$ para realizar a refrigeração do sistema.

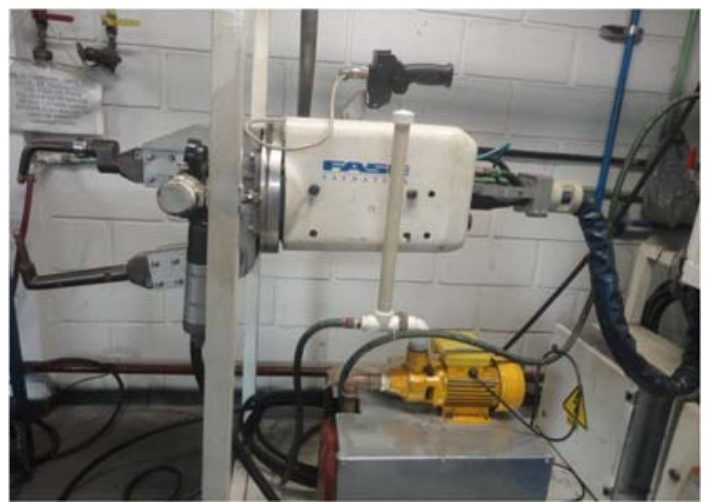

Figura 6. Equipamento utilizado para realização das soldas: FASE, modelo X/4-200.

Para a avaliação do comportamento das chapas em diferentes temperaturas, foi utilizado um sistema de aquisição de dados e os termopares tipo $\mathrm{K}$ soldados aos eletrodos através de descarga capacitiva conforme a Figura 7.

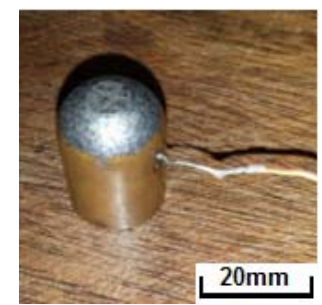

Figura 7. Eletrodo utilizado com termopar soldado por descarga capacitiva. 
Uma das formas de medição da qualidade foi a quantificação das indentações de cada ponto produzido, ou seja, a profundidade máxima dos pontos, para isso foi utilizado um relógio comparador com resolução de $0,01 \mathrm{~mm}$ da marca Mitutoyo modelo ETX964.

\subsection{Métodos}

As chapas foram preparadas nas dimensões propostas pela norma e separadas em pares que seriam soldados para obtenção do corpo de prova.

Os pontos não foram adquiridos de forma consecutiva, e a soldagem só foi realizada após a estabilização da temperatura indicada no sistema de aquisição, e a temperatura importante no processo, e que foi monitorada, foi a temperatura do eletrodo. A Figura 8 mostra um exemplo de uma das curvas obtidas pelo sistema de aquisição para uma temperatura do eletrodo de $40^{\circ} \mathrm{C}$ no momento da soldagem.

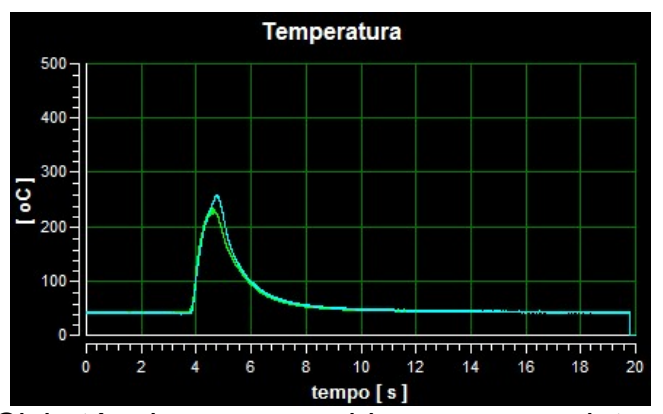

Figura 8. Ciclo térmico para a soldagem como eletrodo à $40^{\circ} \mathrm{C}$.

A corrente de soldagem foi fixada em 4,5kA e o tempo de soldagem foi dividido em: 16 ciclos na compressão, 14 ciclos durante a soldagem, 2 ciclos durante a retenção e 8 ciclos durante a pausa. A força entre eletrodos foi de $360 \mathrm{kgf}$. Estes parâmetros foram mantidos constantes durante todos os ensaios, objetivando avaliar 0 desempenho dos diferentes valores de temperatura em condições semelhantes de operação.

De posse de todos os corpos de prova soldados, foram feitas medições das indentações utilizando o relógio comparador, para diminuir a incerteza das medições, foi realizado uma média dos valores obtidos em 4 pontos opostos e equidistantes em relação ao centro do ponto, a região das medições pode ser observada na Figura 9:

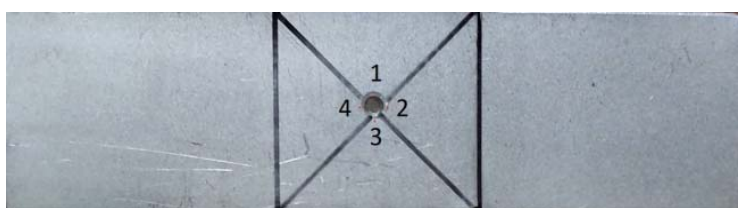

Figura 8.Corpo de prova soldado, evidenciado locais das medições de indentação.

Cada corpo de prova tinha dois lados, que foram nomeados: "Lado A" e "Lado B", o Lado A sempre ficava voltado para o lado da pinça onde ocorre o movimento pneumático durante a soldagem. Depois de feitas as medidas de indentação os corpos de prova foram cortados na região do ponto de solda, para então serem embutidos em baquelite para realização posterior dos testes de dureza e análise metalográfica. O esquema da região de corte pode ser visto na Figura 9. 


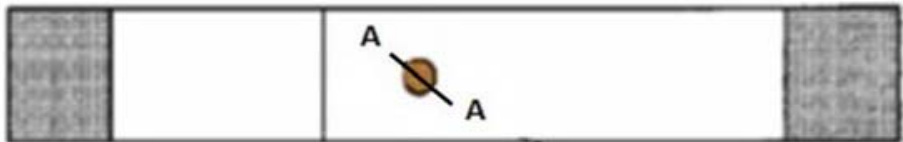

Figura 9. Esquema da região de corte A-A para realização das análises metalográficas.

Após embutimento os pontos de solda foram lixados até uma lixa com granulometria de 1200 e posteriormente polidas com alumina de 0,3 $\mu$ m para então serem atacadas com Nital $5 \%$ para a análise metalográfica, sendo utilizado um microscópio OLYMPUS modelo BX60M com aumento de 10, 20 e 50 vezes. Junto ao microscópio existe uma câmera SONY modelo DXC-151A para captura das imagens. Após as peças serem atacadas e limpas analisou-se qualitativamente. As imagens feitas são mostradas, evidenciando a lente produzida e a região do "centro do cordão/Zona Termicamente Afetada/Metal de base".

Depois de feitas as imagens no microscópio, realizou-se o ensaio de microdureza nos corpos de prova selecionados. Os pontos de indentação foram posicionados como mostrado na Figura 10.

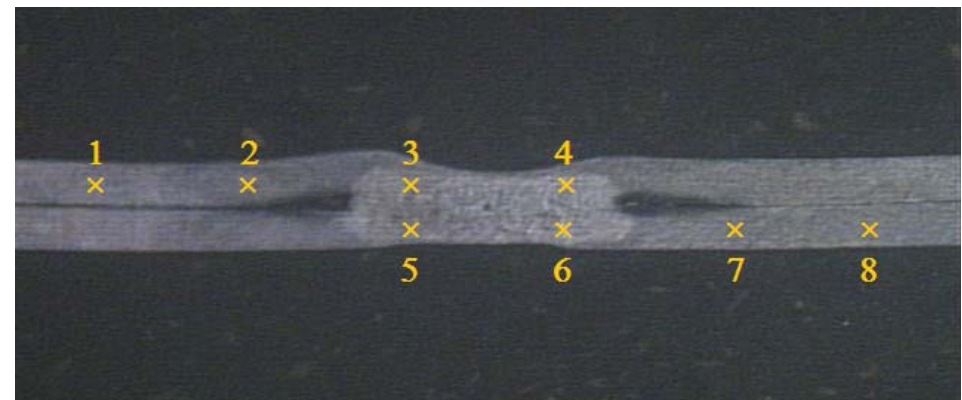

Figura 10. Corpo de prova soldado. Evidenciadas as regiões de indentação para teste de dureza.

A microdureza, medida em Vickers, com carga de 0,20kgf por 15 segundos.

\section{RESULTADOS E DISCUSSÃO}

\section{1- Análise visual e macrográfica}

Analisando os corpos de prova é perceptível o aumento da largura do ponto de solda. Com o aumento da temperatura também é notado o crescimento da zona dendrítica. Fato este que se deve à uma maior proximidade dos eletrodos quando a pinça está aquecida, reduzindo a resistência elétrica do circuito provocando um aumento de corrente e um maior aquecimento na região de formação dos pontos de solda. As Figuras 11, 12 e 13 evidenciam essa diferença de espessura nos "Nuggets", além disso, na Figura 13 onde se tem o aumento de 50x do corpo de prova soldado com o eletrodo a $50^{\circ} \mathrm{C}$, percebe-se o surgimento de microtrincas superficiais (em destaque), consequência também de um superaquecimento e achatamento excessivo da superfície. 


\subsubsection{Ponto de solda com eletrodo a $30^{\circ} \mathrm{C}$}
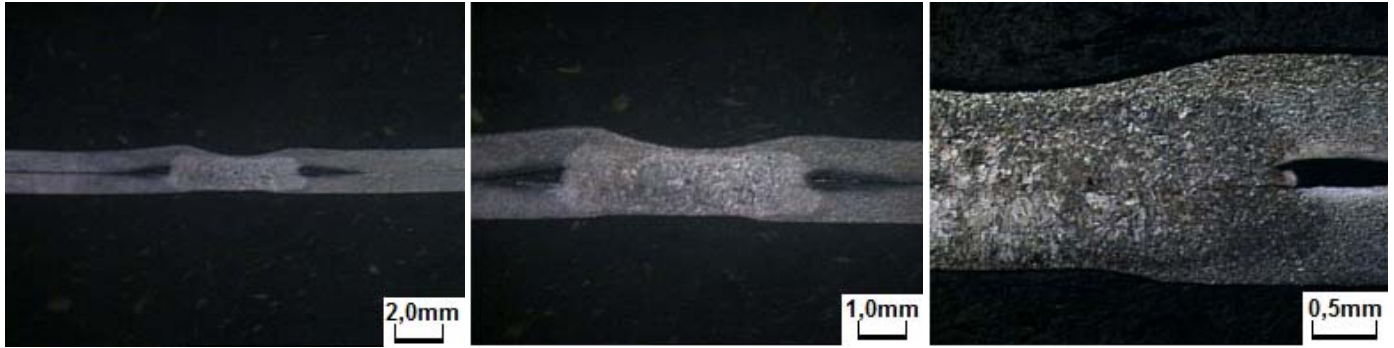

Figura 11. Aumento de $10 \mathrm{x}, 20 \mathrm{x}$ e $50 \mathrm{x}$ em um ponto de solda feito a $30^{\circ} \mathrm{C}$

\subsubsection{Ponto de solda com eletrodo a $40^{\circ} \mathrm{C}$}
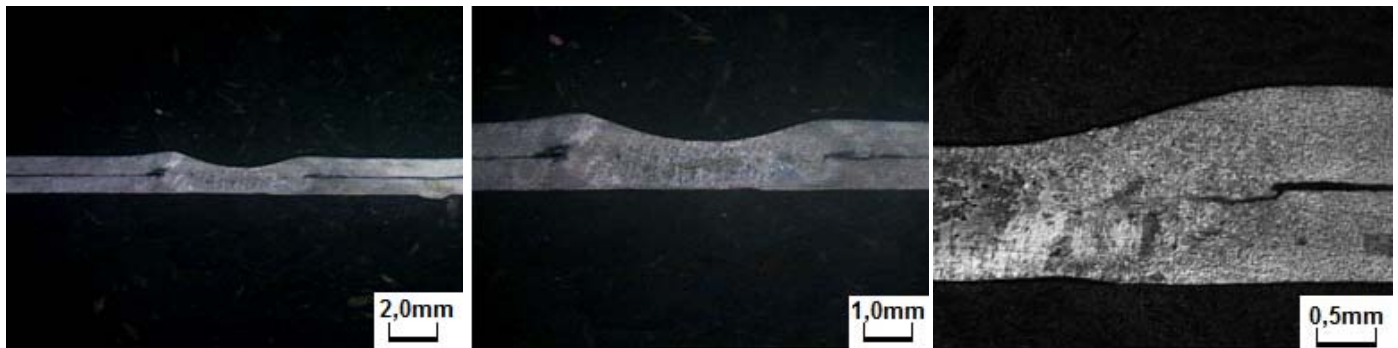

Figura 12. Aumento de 10x, 20 e $50 x$ em um ponto de solda feito com eletrodo a $40^{\circ} \mathrm{C}$.

\subsubsection{Ponto de solda com eletrodo a $50^{\circ} \mathrm{C}$}
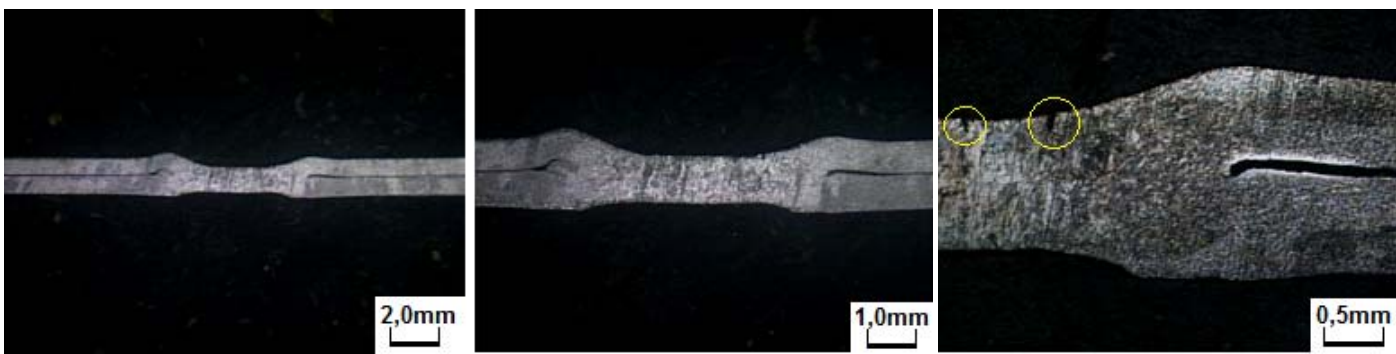

Figura 13. Aumento de $10 \mathrm{x}, 20$ e $50 \mathrm{x}$ em um ponto de solda feito com eletrodo a $50^{\circ} \mathrm{C}$.

\section{2 - Teste de Dureza}

Os resultados para as medidas de microdureza são apresentados na tabela abaixo:

Tabela 1: Valores da microdureza para os pontos de solda em cada temperatura e região. Os

\begin{tabular}{|c|c|c|c|c|c|c|c|c|}
\cline { 2 - 9 } \multicolumn{1}{c|}{} & \multicolumn{8}{c|}{ números das indentações respeitam a ordem apresentada na figura 12.} \\
\hline CP & $\mathbf{1}$ & $\mathbf{2}$ & $\mathbf{3}$ & $\mathbf{4}$ & $\mathbf{5}$ & $\mathbf{6}$ & $\mathbf{7}$ & $\mathbf{8}$ \\
\hline $30 \circ \mathrm{C}$ & 122 & 143 & 235 & 220 & 204 & 215 & 130 & 122 \\
\hline $40 \circ \mathrm{C}$ & 140 & 133 & 245 & 241 & 234 & 230 & 126 & 129 \\
\hline $50 \circ \mathrm{C}$ & 141 & 133 & 206 & 204 & 198 & 199 & 131 & 124 \\
\hline Posição & \multicolumn{2}{|c|}{ MB Lado A } & \multicolumn{2}{|c|}{ Solda Lado A } & \multicolumn{2}{c|}{ Solda Lado B } & \multicolumn{2}{c|}{ MB Lado B } \\
\hline
\end{tabular}

Todos os valores em Vickers 
Nota-se um aumento nos valores da dureza na região dos pontos de solda em relação ao metal de base, o que já era esperado, mas para uma mesma temperatura há uma maior dureza no "Lado A" em comparação ao "Lado B", o que é acarretado pela maior deformação plástica sofrida pelo primeiro no momento da soldagem, pois é o lado onde tem-se a pinça móvel e essa realiza o movimento de fechamento sendo o local de aplicação da carga dinâmica.

Além disso, ocorre uma diminuição significativa para os valores da dureza na região dos pontos de solda (indetações $3,4,5$ e 6) em corpos de prova soldados à $50^{\circ} \mathrm{C}$ em relação aos realizados à $30^{\circ} \mathrm{C}$ e $40^{\circ} \mathrm{C}$, apontando uma perda das propriedades mecânicas quando o ponto é realizado com eletrodos a esta temperatura.

\section{3- Parâmetros geométricos}

Os valores médios para indentação de cada ponto de solda são mostrados na Tabela 2, nesse momento vale ressaltar que a indentação é considerada a medida do ponto mais baixo na região de soldagem até a altura da chapa não deformada.

Tabela 2: Valores das medições de profundidade das indentações para cada corpo de prova.

\begin{tabular}{|c|c|c|c|c|c|c|c|}
\hline $\begin{array}{l}\text { Temp. } \\
\text { eletrodo }\end{array}$ & $\begin{array}{c}\text { no do } \\
\text { CP }\end{array}$ & $\begin{array}{c}\text { Lado } \\
\text { medido }\end{array}$ & $\begin{array}{c}\text { medida } \\
1\end{array}$ & $\begin{array}{c}\text { medida } \\
2\end{array}$ & $\begin{array}{c}\text { medida } \\
3\end{array}$ & $\begin{array}{c}\text { medida } \\
4\end{array}$ & $\begin{array}{l}\text { Valor médio das } \\
\text { indentações }\end{array}$ \\
\hline \multirow{6}{*}{$30 \circ \mathrm{C}$} & \multirow{2}{*}{$\mathrm{CP} 1$} & Lado A & 80,0 & 34,0 & 61,0 & 36,5 & 52,88 \\
\hline & & Lado B & 58,0 & 45,0 & 57,0 & 38,5 & 49,63 \\
\hline & \multirow{2}{*}{$\mathrm{CP} 2$} & Lado A & 25,0 & 71,0 & 41,0 & 62,0 & 49,75 \\
\hline & & Lado B & 26,0 & 45,0 & 35,5 & 39,0 & 36,38 \\
\hline & \multirow{2}{*}{$\mathrm{CP} 3$} & Lado A & 65,0 & 20,0 & 51,0 & 26,0 & 40,50 \\
\hline & & Lado B & 39,0 & 45,5 & 46,0 & 52,0 & 45,63 \\
\hline & & & & & valor con & iderado & 45,79 \\
\hline \multirow{6}{*}{$40 \circ \mathrm{C}$} & \multirow{2}{*}{$\mathrm{CP} 1$} & Lado A & 62,0 & 38,0 & 56,0 & 43,0 & 49,75 \\
\hline & & Lado B & 38,0 & 38,0 & 39,0 & 41,0 & 39,00 \\
\hline & \multirow{2}{*}{$\mathrm{CP} 2$} & Lado A & 42,5 & 52,0 & 61,0 & 37,0 & 48,13 \\
\hline & & Lado B & 28,0 & 60,0 & 25,0 & 65,0 & 44,50 \\
\hline & \multirow{2}{*}{$\mathrm{CP} 3$} & Lado A & 58,0 & 27,0 & 49,5 & 42,0 & 44,13 \\
\hline & & Lado B & 25,5 & 37,5 & 68,5 & 49,0 & 45,13 \\
\hline & & & & & valor con & iderado & 45,10 \\
\hline \multirow{6}{*}{$50^{\circ} \mathrm{C}$} & \multirow{2}{*}{$\mathrm{CP} 1$} & Lado A & 65,0 & 30,0 & 48,0 & 41,5 & 46,13 \\
\hline & & Lado B & 23,0 & 52,0 & 30,0 & 53,0 & 39,50 \\
\hline & \multirow{2}{*}{$\mathrm{CP} 2$} & Lado A & 69,5 & 35,0 & 60,5 & 35,0 & 50,00 \\
\hline & & Lado B & 20,0 & 38,5 & 23,5 & 45,0 & 31,75 \\
\hline & \multirow{2}{*}{$\mathrm{CP} 3$} & Lado A & 59,0 & 42,0 & 41,0 & 66,5 & 52,13 \\
\hline & & Lado B & 33,5 & 23,5 & 60,0 & 8,5 & 31,38 \\
\hline
\end{tabular}

*Todos os valores com ordem de $10^{-5} \mathrm{~m}$.

Pelos valores medidos, há uma diminuição nos valores médios de indentação com o aumento da temperatura, em contrapartida do que acontece com a largura do ponto, como já foi discutido. $O$ aumento da largura e redução da indentação média se dá pelo fato do eletrodo, quando trabalhando com maiores temperaturas, sofrer um maior grau de amolecimento, ou seja, ele achata mais quanto mais quente ele está, gerando assim o padrão encontrado.

Com os resultados compilados e analisados, fez-se um teste com um padrão de múltiplos pontos para levantamento de um ciclo térmico para um eletrodo 
refrigerado, ele é apresentado na Figura 14 e mostra a importância de um estudo para a qualidade de soldas realizadas com temperaturas elevadas, uma vez que o este padrão é o que normalmente se encontra em linhas de produção. Na mesma figura tem-se o início da soldagem do primeiro ponto (1) com o eletrodo à $40^{\circ} \mathrm{C}$ e os seguintes ( 2 a 7 ) iniciam a solda com uma temperatura acima de $60^{\circ} \mathrm{C}$. Isso mostra que o valor definido no começo do processo de soldagem pode não se manter quando realizado múltiplos pontos pelo fato do sistema de refrigeração não possuir uma troca térmica suficiente para retornar à situação inicial, fazendo os pontos consecutivos serem produzidos com o eletrodo sobreaquecido e gerando padrões indesejados para a solda como encontrados no trabalho.

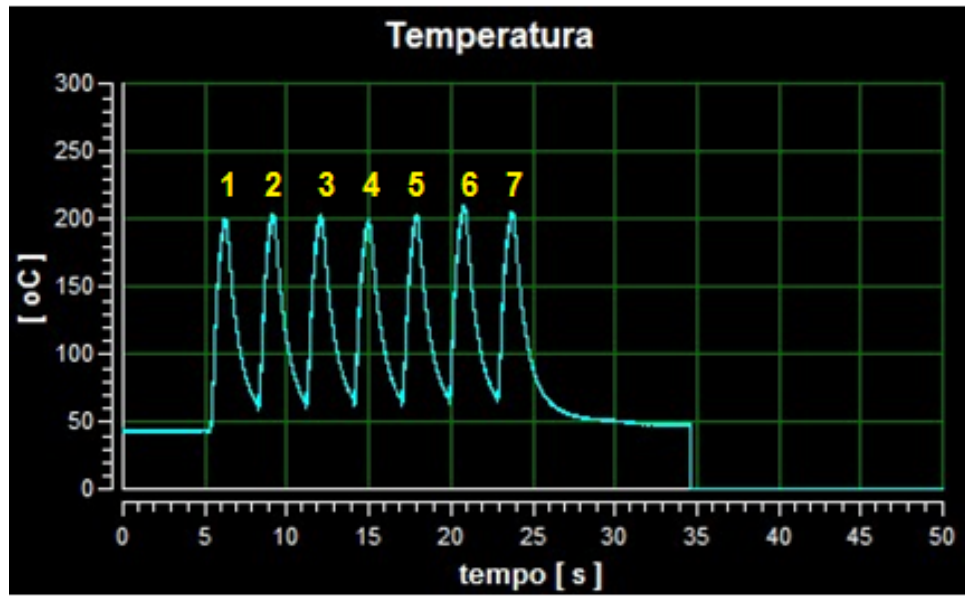

Figura 14. Ciclo térmico de um eletrodo realizando múltiplos pontos e com refrigerção de $40^{\circ} \mathrm{C}$

Com este trabalho é possível ter algumas metas para trabalhos futuros, tais como a implementação de um sistema com melhor refrigeração para otimização da troca térmica no eletrodo, aumentando sua vida útil e produzindo soldas com melhores propriedades.

\section{CONCLUSÕES}

Fazendo o levantamento com todas as informações obtidas através de medições, inspeções visuais, pelos valores de dureza e análise da geometria dos pontos de solda, pode-se concluir que com o aumento da temperatura do eletrodo no momento da soldagem tem-se:

- Aumento da largura do ponto de solda.

- Diminuição dos valores médios para as indentação do ponto de solda.

- Maior amolecimento do eletrodo e consequentemente menor vida útil do mesmo.

- Surgimento de microtrincas nas superfícies dos pontos produzidos.

- Diminuição da dureza do "nugget".

Pode-se também listar que:

- Não há diferenças significativas, dentro da faixa medida, para os ciclos térmicos de aquecimento e resfriamento dos eletrodos.

- As chapas que estão em contato com as pinças móveis sofrem maior deformação plástica no processo. 


\section{Agradecimentos}

\section{Os autores agradecem ao Laboratório de Robótica Soldagem e Simulação (LRSS) da UFMG por disponibilizar os recursos necessários para a realização desta pesquisa.}

\section{REFERÊNCIAS}

1 GIUNTI SALDATI, Saldatura a Pressione, Fiat Group Automobiles Normazione 00920/02, Torino, 2007

2 AWS - American Welding Society, Jefferson`s Welding Enciclopedia. 18th Edition. Miami: 1997. p. 430

3 KEARNS, W. H. Welding Handbook: Resistance and Solid-State Welding and Other Joining Processes. Miami: American Welding Society, 1980, 7. Vol. 3.

4 CHANG, H. S. e CHO, H. S., 1990, "A Study on the Shunt Effect in Resistance Spot Welding”, Welding Journal, August, Vol. 69, N8, pp. 308-317.

5 MARQUES, P. V. MODENESI, P. J. BRACARENSE, A. Q., 2009, "Soldagem: Fundamentos e Tecnologia”. 3 ed. Belo Horizonte: Editora UFMG, 2009. 363p.

6 BRANCO, H. L. O., "Avaliação de capas de eletrodos utilizadas na soldagem por resistência de chapas galvanizadas automotivas", Belo horizonte, Brasil

7 COTA, S. B., "Metodologia para ensaio da resistência à torção de solda a ponto por resistência elétrica", Belo Horizonte, Brasil. 2015:60-85

8 STOCCO, D., GONÇALVES, R., 2003, "Caracterização dos Defeitos Encontrados em Solda a Ponto Utilizando Técnica do Ultra-Som, Apoiado a Exames Metalográficos", 3rd Pan American Conference for Nondestructive Testing, Hotel Inter Continental, Rio de Janeiro, RJ, Brazil.

9 POWELL, H. J., Westgate S. A.,Wiemer, K., A pratical guide to process and quality control for resistance spot welding, The Welding Institute, 1992.

10 AMERICAN WELDING SOCIETY - AWS., 1998, Welding Handbook. Vol. 2: Welding Process, $8^{\circ}$ ed. International Standard Book Number: 0-87171-354-3. American Welding Society. 1998. 550 N. W. LeJeune Rd., P.O. Box 351040, Miami, FL 33135.

11 AWS/SAE D8.9M., 2002, "Recommended Practices for Test Methods for Evaluating the Resistance Spot Welding Behavior of Automotive Sheet Steel Materials". International Standard Book Number: 0-87171-672-0. 2002. American Welding Society. 550 N. W. LeJeune Road, Miami, Florida 33126. Printed in the U.S.A. 